\title{
Correlation between semen examination based on the WHO criteria and sperm DNA fragmentation test
}

Tsuyoshi Okubo ( $\nabla$ t-okubo@yumeclinic.net)

Shinbashi Yume Clinic https://orcid.org/0000-0002-6915-3633

\section{Noriyuki Onda}

Shinbashi Yume Clinic

\section{Teruaki Hayashi}

Shinbashi Yume Clinic

\section{Kenji Omi}

Shinbashi Yume Clinic

\section{Tomoya Segawa}

Shinbashi Yume Clinic

\section{Research article}

Keywords: Semen examination, Sperm DNA fragmentation, Sperm morphology, Infertility of male, Spermatogenesis

Posted Date: May 3rd, 2021

DOI: https://doi.org/10.21203/rs.3.rs-189841/v2

License: (c) (i) This work is licensed under a Creative Commons Attribution 4.0 International License. Read Full License 


\section{Abstract}

In this study, we performed and analyzed the DFI test and semen test based on the WHO criteria and compared the two using semen factors. DFI tests and general semen tests were conducted in accordance with the WHO criteria, and correlations between the two tests were investigated. The WHO criteria were set as the cutoff value for each of the following factors: fluid volume, concentration, motility, and normal morphology, and compared with the DFI results. The subjects had a mean DFI of $15.6 \pm 11.5 \%$ and the DFI increased with age. In contrast, the concentration, motility, and normal morphology decreased as the DFI increased. Patients who satisfied the WHO criteria in terms of sperm concentration, motility, and normal morphology had a significantly lower DFI than the group that did not satisfy the criteria. Therefore, evaluation with a general semen test based on the WHO criteria should be regarded as a qualitative evaluation of all factors other than fluid volume. Male infertility due to sperm DNA fragmentation should be suspected when in vitro fertilization (IVF) results are poor despite normal semen findings based on the WHO criteria. The results of this study suggest that the DFI test may be an important evaluation in follow-up semen analyses.

\section{Introduction}

Female factors are very important in determining the success of IVF. However, many reports have shown that male factors also affect the success or failure of IVF (1) (2). Thus, male factors, analyzed via semen tests, are important (3). Generally, the tests are based on diagnostic methods in line with the WHO criteria (4) and investigate factors such as semen fluid volume, concentration, motility, and malformation rate.

The diagnostic sensitivity of semen analysis is low. In other words, it cannot be said that the proportion of sperm in motion alone essentially represents the quality of sperm motility. Evaluation of the malformation rate is also subjective, as there could be individual differences and is based only on an approximate morphological sperm evaluation viewed through a biological microscope. Therefore, the sperm evaluation criteria based on conventional methods alone is not a primary factor of consideration for determining IVF embryonic potential, pregnancy rates, and miscarriage rates after transplantation.

In our hospital, we evaluate sperm motility using an automatic sperm analyzer based on the WHO criteria and sperm morphology via the Kruger test (5).

In addition to evaluating the number and motility of sperms, factors that contribute to infertility, such as whether the sperms have the ability to fertilize oocytes and how they are involved in embryogenesis after fertilization, should be evaluated. Therefore, we considered the DFI (6-8). The DFI is based on a sperm chromatin nucleus analysis (9). The aim of the DFI is to evaluate qualitative factors of the sperm nuclei. Methods for detecting DFI include TUNEL assay (fluorescence microscopy with terminal deoxynucleotidyl transferase-mediated deoxyuridine triphosphate-nick end labeling), comet assay (detection with singlecell gel electrophoresis), and AO test (fluorescence microscopy using acridine orange, a nucleic acid fluorescent dye). However, these DFI analysis techniques require sophisticated equipment and are expensive. In this study, the DFI analysis was performed using the sperm chromatin dispersion (SCD) test 
based on chromatin structural analysis of the sperm nucleus (10) and quantified via the Halo sperm DNA kit (Spain, Madrid).

This kit is commercially available and enables relatively simple and low cost DFI analysis.

The protamine in sperm nuclei with less DNA fragmentation forms a halo in DNA strands extracted using the acid and detergent treatment. The principle of measurement utilizes the property that halo formation is inhibited in DFI fragmented sperm nuclei. DFI is calculated as the number of non-halo-forming sperms of the total number of sperms and expressed as a percentage (11).

We considered various findings and evaluated the usefulness of the DFI test against WHO criteria, using the DFI test results of patients who visited our hospital for infertility treatment.

\section{Subjects And Methods}

Study population

This study was conducted from June 2020 to December 2020. A total of 134 male patients with the main complaint of infertility who requested semen testing were included. All patients provided written consent. The study was approved by the ethics review board of our hospital. The tests included in the study were the automatic sperm motility analysis using SMAS, Kruger test via the Diff-quick method, and DFI test using the SCD method.

Semen examination and analysis

¿Sperm motility test (general semen test)

The fluid volume was measured after the semen was sufficiently liquified, and the sperm concentration and motility were measured with SMAS (Japan, DETECT).

¿Sperm morphology (Kruger Sperm Function Test)

The sperms were treated using the Diff-quick staining kit (Sysmex Corporation, Japan). Normal morphology was analyzed using 200 or more sperms under a biological microscope $(\times 400)$, and the head, midpiece, and tail of the sperms were examined in detail.

¿Sperm DNA fragmentation test (DFI test)

DFI evaluation was conducted using the Halo sperm DNA kit (HT-HS10, Halotech DNA, Spain). It is possible to quantify DNA fragmentation in the sperm nucleus using this kit. In normal sperms, halos formed by the loop strands of DNA in the head are visible, but halos do not form in the loop strands of damaged DNA of fragmented sperms. The ratio of fragmented sperms to the total number of sperms was analyzed and expressed as the DFI ratio using a selection of 300 or more sperms. 
We investigated the correlation between the DFI tests and semen analyses based on the WHO 2010 criteria using the following factors: semen fluid volume, sperm concentration, total sperm count, motility, and normal morphology. In addition, we also compared each factor by separating the subjects into two groups: the group with measurements below the WHO criteria and the group with measurements at or above the WHO criteria.

\section{Results}

The mean age of the 134 patients at the time of the sperm tests was $38.8 \pm 6.0$ years (range $=26-52$ years). All the patients were examined at our hospital for the first time. Semen fluid volume, sperm concentration, total sperm count, and motility were investigated using the general semen test. Normal morphology was examined via the Kruger Test. The DFI ratio was investigated using the DFI test (Table 1).

Table 1

General semen findings and DFl in target patients

\begin{tabular}{|lll|}
\hline Factors & Mean \pm SD & Range \\
\hline Age (years) & $38.8 \pm 6.0$ & $26-52$ \\
\hline Semen fluid volume $(\mathrm{mL})$ & $2.8 \pm 1.4$ & $0.3-9.4$ \\
\hline Sperm concentration $\left(10^{6} / \mathrm{mL}\right)$ & $76.5 \pm 60.1$ & $0.5-284.0$ \\
\hline Sperm motility $(\%)$ & $63.5 \pm 19.8$ & $0-93.2$ \\
\hline Normal morphology $(\%)$ & $3.2 \pm 2.0$ & $0-10.0$ \\
\hline DFI (\%) & $15.6 \pm 11.5$ & $2.4-97.0$ \\
\hline
\end{tabular}

The mean patient DFI was $15.6 \pm 11.5 \%$. Using the Halo sperm HT-HS10 DNA kit per instructions provided in the package insert, $6.0 \%$ (8/134) of the patients had high DFI ( $\geq 30 \%)$ (Fig. 1).

There was tendency for the DFI to increase with age $(r=0.2319, p<0.0070)$, while the concentration, motility, and normal morphology tended to decrease as the DFI increased (Table 2). 
Table 2

Various factors in general semen tests and correlation with

DFI

\begin{tabular}{|lll|}
\hline & DFI & \\
\hline Factor & Correlation $(r)$ & P value \\
\hline Age (years) & 0.2319 & 0.0070 \\
\hline Semen fluid volume $(\mathrm{mL})$ & -0.0118 & 0.8926 \\
\hline Sperm concentration $\left(10^{6} / \mathrm{mL}\right)$ & -0.1992 & 0.0210 \\
\hline Sperm motility $(\%)$ & -0.2672 & 0.0018 \\
\hline Normal morphology $(\%)$ & -0.2881 & 0.0007 \\
\hline
\end{tabular}

A comparative examination of the DFI against the WHO criteria did not reveal a significant difference between the group at or above the criteria and the group below the criteria for fluid volume $(p=0.6891)$. However, for concentration, motility, and normal morphology, those below the criteria (that is, those which did not meet the WHO criteria) tended to have higher DFI compared to that of the group at or above the criteria (concentration $p<0.05$; motility $p<0.01$; normal morphology $p<0.01$ ). Moreover, when these factors were above the criteria, the DFI was evaluated to be low for all factors (Fig. 2). When the WHO criteria were not met, the DFI was determined to be moderate or high for all factors.

\section{Discussion}

Using the DFI evaluation for semen tests enabled clarification of the correlation between DFI and various factors that are considered to be related to the performance outcome of assisted reproductive technologies.

There are still many unknown aspects of the causes of infertility that are related to male factors rather than female factors. Generally, semen tests used for evaluation and diagnostics focus primarily on semen fluid volume, sperm concentration, motility, and malformation rate.

The information obtained in these general semen test findings are considered inadequate for determining sperm potential. To more accurately evaluate the correlation between IVF clinical outcomes and male infertility, we focused on DFI measurements.

DFI is a method for detecting the integrity of sperm nuclear DNA and the extent of damage. DFI is reported to increase with age in men. Reports have shown that close to $8 \%$ of infertile men have high DFI values ( $\geqq 30 \%$ ) (12). Reports have also suggested that factors associated with sperm DNA damage are affected by abnormal sperm lipids, reproductive hormones, and mitochondria (13). These factors are involved in oxidative stress and apoptosis formation due to an age-dependent decline in male fecundity. Therefore, causing an age-related increase in DNA damage (14). 
The results of our DFI measurements in this study showed that DFI tended to increase with age, as described in previous reports (15-17). However, DFI is not only affected by age, but also by indulgences such as cigarettes (18) and alcohol (19), lifestyle habits such as sleep and exercise, and intake of supplements (20). Thus, various aspects of aging, multiple stress factors, and negative factors affecting the DFI require further investigations (21). In terms of the link with IVF outcomes, it has been reported that, although there is no difference in the fertilization rate and embryonic development rate between high DFI and low DFI (22), a high DFI tends to be associated with higher rates of miscarriage, resulting in a low live birth rate per transplantation $(23,24)$. The patients who underwent DFI analysis in this study provided semen samples before IVF treatment. The mean DFI was $15.6 \pm 11.5 \%$. Generally, DFI levels of $\geq 30 \%$ are considered to be high, and therefore, it is necessary to investigate the correlation between elevated DFI and IVF outcomes.

We also found that DFI was significantly lower in the group below the criteria compared to the group at or above the criteria for sperm concentration, motility, and normal morphology, in semen tests conducted in accordance with the WHO criteria (25). The results suggest that, when the WHO criteria test results were good, DFI evaluation was also generally good (26). However, it is necessary to suspect damage to the sperm nucleus when IVF outcomes are poor despite patients satisfying the WHO criteria, and DFI analysis may be useful as an additional semen test in these circumstances.

\section{Conclusion}

In conclusion, DFI analysis may lead to elucidation of potential factors for predicting IVF outcomes. Incorporating the DFI into standard semen analysis may result in a more reliable semen diagnostic technique.

In the future, further techniques to evaluate the contribution of sperm DNA to IVF outcomes and for the selection of the best sperms, will lead to improved IVF outcomes. Even during the IVF process, certain factors may lead to an increase in $\mathrm{DFI}$, including active oxygen in the semen (27), the sperm screening method (28-30), and culturing conditions $(31,32)$. Therefore, we would like to investigate whether optimal sperm selection techniques are possible by reducing the external stress factors.

\section{Declarations}

\section{Conflict of interests}

The authors declare no conflict of interest.

\section{Ethical approval and consent to participate}

This study was approved by the Institutional Review Board of Shinbashi Yume Clinic. This is a retrospective study in patients who submitted informed consent for undergoing fertility treatment at our IVF center. 


\section{Consent for publication}

Not applicable.

\section{Availability of data and materials}

Please contact author for data requests.

\section{Authors' contributions}

TO: project development, data collection, data analysis and manuscript writing. NO and TH: data collection and data analysis KO: contributed to interpretation and writing. TS: contributed to interpretation, writing and manuscript revising. All authors reviewed and approved the final manuscript.

\section{Acknowledgement}

Not applicable.

\section{Funding}

No funding was received.

\section{Abbreviations}

WHO: World Health Organization; DFI: Sperm DNA Fragmentation Index; IVF: In Vitro Fertilization;

SCD: sperm chromatin dispersion

\section{References}

1. Softness KA, Trussler JT, Carrasquillo RJ. Advanced sperm testing. Curr Opin Urol. 2020;30(3):290-5.

2. Lopez G, Lafuente R, Checa MA, Carreras R, Brassesco M. Diagnostic value of sperm DNA fragmentation and sperm high-magnification for predicting outcome of assisted reproduction treatment. Asian J Androl. 2013;15(6):790-4.

3. Agarwal A, Mulgund A, Hamada A, Chyatte MR. A unique view on male infertility around the globe. Reprod Biol Endocrinol. 2015;13:37.

4. Christopher L R Barratt, Lars Björndahl, Christopher J De Jonge, Dolores J Lamb, Francisco Osorio Martini, Robert McLachlan, Robert D Oates, Sheryl van der Poel, Bianca St John, Mark Sigman, Rebecca Sokol, Herman Tournaye. The diagnosis of male infertility: an analysis of the evidence to support the development of global WHO guidance-challenges and future research opportunities. Hum Reprod Update. 2017;23(6):660-80.

5. T F Kruger, A A Acosta, K F Simmons, R J Swanson, J F Matta, L L Veeck, M Morshedi, S Brugo. New method of evaluating sperm morphology with predictive value for human in vitro fertilization. 
Urology. 1987;Sep;30((3)):248-51.

6. Jin-Chun Lu, Jun Jing, Li Chen, Yi-Feng Ge, Rui-Xiang Feng, Yuan-Jiao Liang, Bing Yao. Analysis of human sperm DNA fragmentation index (DFI) related factors: a report of 1010 subfertile men in China. Reprod Biol Endocrinol. 2018;16(1):23.

7. Li MW, Lloyd KCK. DNA fragmentation index (DFI) as a measure of sperm quality and fertility in mice. Sci Rep. 2020;10(1):3833.

8. Yang H, Li G, Jin H, Guo Y, Sun Y. The effect of sperm DNA fragmentation index on assisted reproductive technology outcomes and its relationship with semen parameters and lifestyle. Transl Androl Urol. 2019;8(4):356-65.

9. Wiweko B, Utami P. Predictive value of sperm deoxyribonucleic acid (DNA) fragmentation index in male infertility. Basic Clin Androl. 2017;27:1.

10. Claudia G Petersen, Ana L Mauri, Laura D Vagnini, Adriana Renzi, Bruna Petersen, Mariana Mattila, Vanessa Comar, Juliana Ricci, Felipe Dieamant, Joao Batista A Oliveira, Ricardo L R Baruffi, Jose G Franco Jr. The effects of male age on sperm DNA damage: an evaluation of 2,178 semen samples. JBRA Assist Reprod. 2018;22(4):323-30.

11. M Tandara, A Bajić, L Tandara, L Bilić-Zulle, M Šunj, V Kozina, T Goluža, M Jukić. Sperm DNA integrity testing: big halo is a good predictor of embryo quality and pregnancy after conventional IVF. Andrology. 2014;2(5):678-86.

12. Le MT, Nguyen TAT, Nguyen HTT, Nguyen TTT, Nguyen VT, Le DD, Nguyen VQH, Cao NT. Does sperm DNA fragmentation correlate with semen parameters? Reprod Med Biol. 2019;18(4):390-6.

13. Agarwal A, Panner Selvam MK, Baskaran S, Cho CL. Sperm DNA damage and its impact on male reproductive health: a critical review for clinicians, reproductive professionals and researchers. Expert Rev Mol Diagn. 2019;19(6):443-57.

14. Rosiak-Gill A GK, Jakubik J, Fraczek M, Patorski L, Gaczarzewicz D, Kurzawa R, Kurpisz M, Piasecka M. Age-related changes in human sperm DNA integrity. Aging. 2019;11(15):5399-411.

15. Deenadayal Mettler A, Govindarajan M, Srinivas S, Mithraprabhu S, Evenson D, Mahendran T. Male age is associated with sperm DNA/chromatin integrity. Aging Male. 2019:1-8.

16. Donald P Evenson. Evaluation of sperm chromatin structure and DNA strand breaks is an important part of clinical male fertility assessment. Transl Androl Urol. 2017;6(Suppl 4):S495-S500.

17. Albani E CS, Gurrieri B, Arruzzolo L, Negri L, Borroni EM, Levi-Setti PE. Male age negative impact on sperm DNA fragmentation. Aging. 2019;11(9):2749-61.

18. Silver EW, Eskenazi B, Evenson DP, Block G, Young S, Wyrobek AJ. Effect of antioxidant intake on sperm chromatin stability in healthy nonsmoking men. J Androl. 2005;26(4):550-6.

19. Shi X, Chan CPS, Waters T, Chi L, Chan DYL, Li TC. Lifestyle and demographic factors associated with human semen quality and sperm function. Syst Biol Reprod Med. 2018;64(5):358-67.

20. Shathmigha Ketheeswaran, Susanne Elisabeth Pors, Lenin Arturo Zuniga Jara, Camilla Engel Lemser, Terese Kimmie Høj, Jane Alrø Bøtkjær, Preben Christensen, Peter Humaidan, Stine Gry Kristensen. 
Effect of whey protein supplementation on sperm quality and fertility in male mice. Food Chem Toxicol. 2020;141:111366.

21. Vinnakota C, Cree L, Peek J, Morbeck DE. Incidence of high sperm DNA fragmentation in a targeted population of subfertile men. Syst Biol Reprod Med. 2019;65(6):451-7.

22. Green KA, Patounakis G, Dougherty MP, Werner MD, Scott RT, Jr., Franasiak JM. Sperm DNA fragmentation on the day of fertilization is not associated with embryologic or clinical outcomes after IVF/ICSI. J Assist Reprod Genet. 2020;37(1):71-6.

23. Lin MH, Kuo-Kuang Lee R, Li SH, Lu CH, Sun FJ, Hwu YM. Sperm chromatin structure assay parameters are not related to fertilization rates, embryo quality, and pregnancy rates in in vitro fertilization and intracytoplasmic sperm injection, but might be related to spontaneous abortion rates. Fertil Steril. 2008;90(2):352-9.

24. Yifu P, Lei Y, Shaoming L, Yujin G, Xingwang Z. Sperm DNA fragmentation index with unexplained recurrent spontaneous abortion: A systematic review and meta-analysis. J Gynecol Obstet Hum Reprod. 2020:101740.

25. Westerman R. Biomarkers for demographic research: sperm counts and other male infertility biomarkers. Biodemography Soc Biol. 2020;65(1):73-87.

26. Colasante A, Minasi MG, Scarselli F, Casciani V, Zazzaro V, Ruberti A, et al. The aging male: Relationship between male age, sperm quality and sperm DNA damage in an unselected population of 3124 men attending the fertility centre for the first time. Arch Ital Urol Androl. 2019;90(4):254-9.

27. C Wright SM, H Leeson. Sperm DNA damage caused by oxidative stress modifiable clinical, lifestyle and nutritional factors in male infertility. Reprod Biomed Online. 2014;28(6):684-703.

28. Tsuyoshi Okubo, Kenta Higuchi, Teruaki Hayashi, Noriyuki Onda, Ryoko Matsuo, Kenji Omi, Tomoya Segawa. Evaluation of motility and DNA fragmentation of sperm purified by microfluidics and Migration-Gravity sedimentation. Journal of Mammalian Ova Research. 2020;37(2):107-11.

29. Shirota K, Yotsumoto F, Itoh H, Obama H, Hidaka N, Nakajima K, Miyamoto S. Separation efficiency of a microfluidic sperm sorter to minimize sperm DNA damage. Fertil Steril. 2016;105(2):315-21 e1.

30. Masaya Yamanaka, Kazuhisa Tomita, Shu Hashimoto, Hiroshi Matsumoto, Manabu Satoh, Hiromi Kato, Yoshihiko Hosoi, Masayasu Inoue, Yoshiharu Nakaoka and Yoshiharu Morimoto. Combination of density gradient centrifugation and swim-up methods effectively decreases morphologically abnormal sperms. Journal of Reproduction and Development. 2016;62(6):599-606.

31. Makkar G NH, Yeung SB, Ho PC. Comparison of two colloidal silica-based sperm separation media with a non-silica-based medium. Fertility and Sterility. 1999;72(5):796-802.

32. Matsuura R, Takeuchi T, Yoshida A. Preparation and incubation conditions affect the DNA integrity of ejaculated human spermatozoa. Asian J Androl. 2010;12(5):753-9.

\section{Figures}




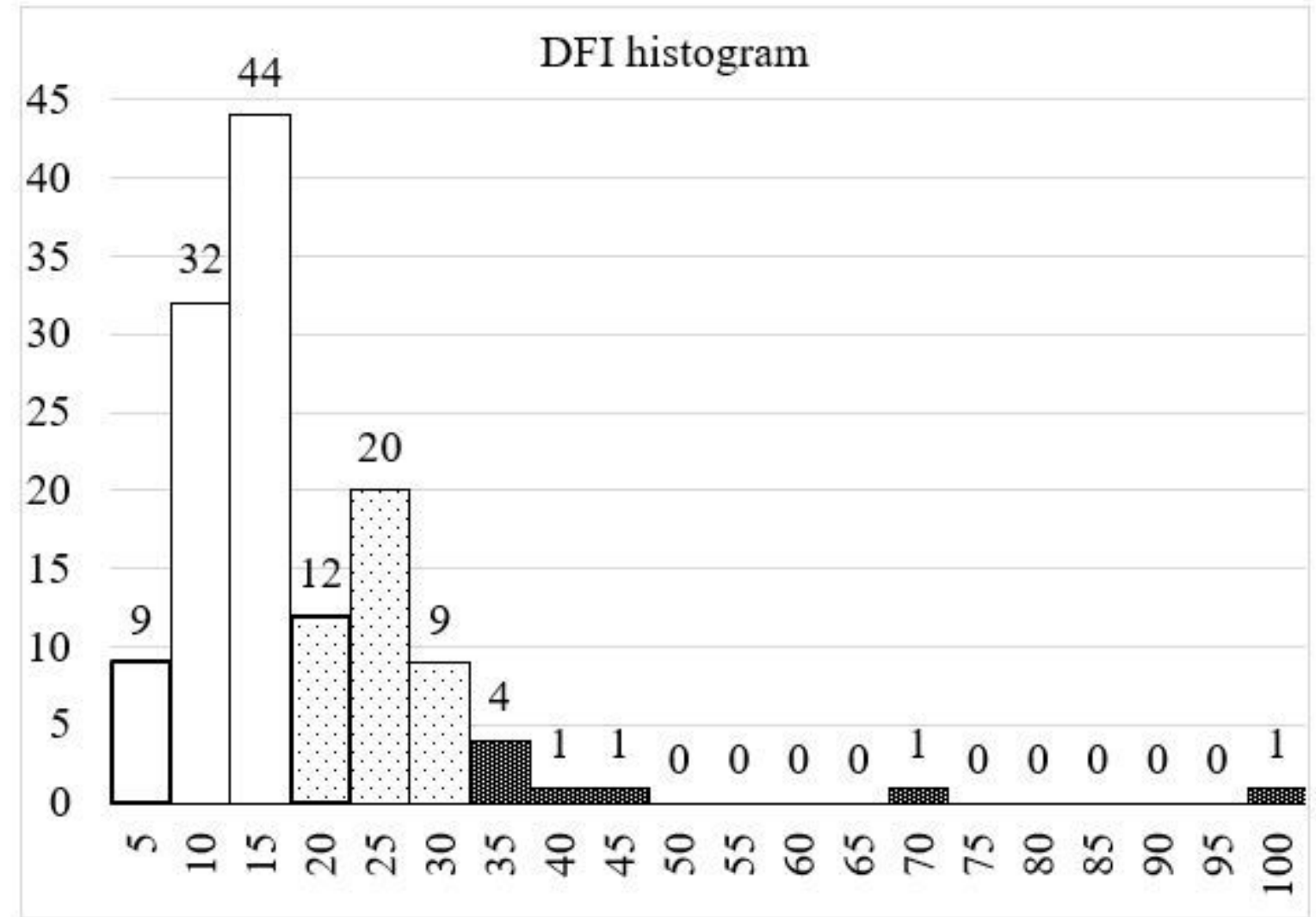

DFI evaluation

$\mathrm{n}$ (people)

Percentage of the

whole (\%)

\begin{tabular}{lcc}
\hline Low $(<15 \%)$ & 85 & 63.4 \\
Moderate $(15-<30 \%)$ & 41 & 30.6 \\
High $(\geq 30 \%)$ & 8 & 6.0 \\
\hline
\end{tabular}

Figure 1

DFI distribution and percentage for each evaluation The mean DFI was $15.6 \pm 11.5 \%$, with normal DFI rating accounting for $63.4 \%$. On the other hand, samples with Moderate and High DFI evaluation accounted for about one-third and more. Especially, the highest DFI was 97.0\% (range; 2.4-97.0). 
FLUID VOLUME

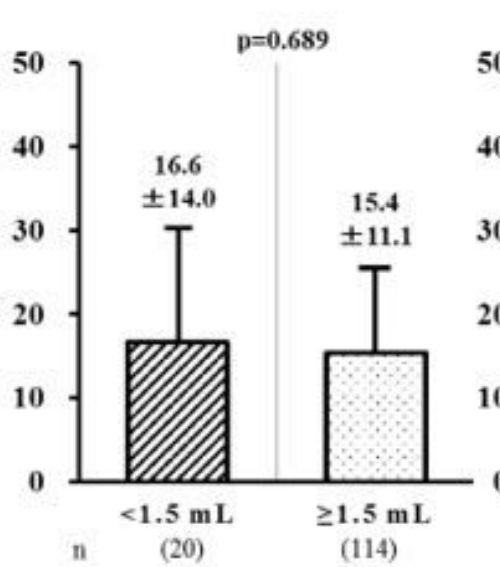

CONCENTRATION

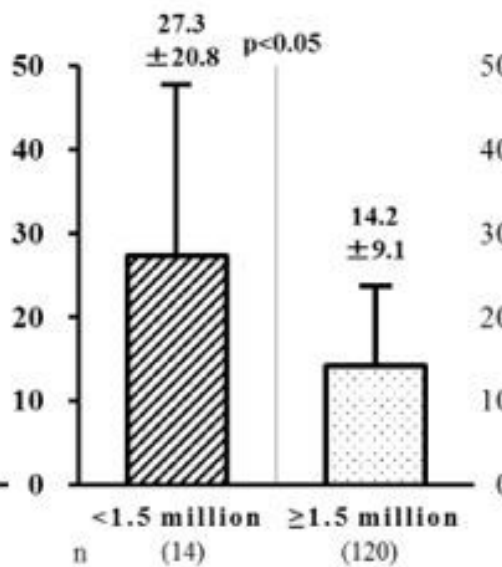

MOTILITY

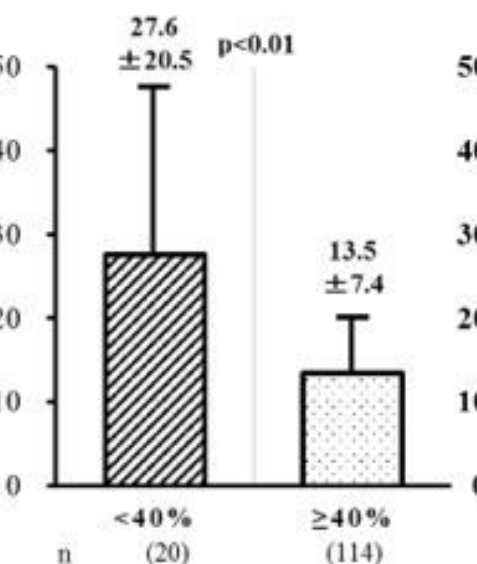

NORMAL MORPHOLOGY

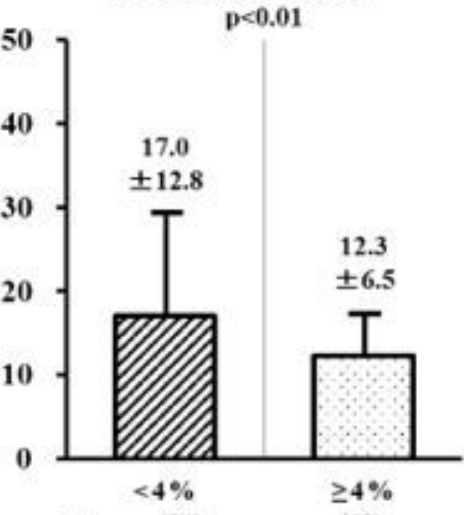

$(40)$

Figure 2

DFI comparison in the group at or above and the group below the WHO criteria for each factor in the general semen test There was no significant difference in DFI for semen fluid volume between the group at or above the criteria and the group below the criteria. Regarding the concentration, motility, and normal morphology, the group below the criteria (that is, the group that did not meet the WHO criteria) had higher DFI. 